\title{
Advances in Biomaterials for the Prevention and Disruption of Candida Biofilms
}

\section{OPEN ACCESS}

Edited by:

Sara María Soto,

Instituto Salud Global Barcelona

(ISGlobal), Spain

Reviewed by:

Renátó Kovács,

University of Debrecen, Hungary

Rodnei Dennis Rossoni,

São Paulo State University, Brazil

Liliana Scorzoni,

São Paulo State University, Brazil

${ }^{*}$ Correspondence:

Anita Shukla

anita_shukla@brown.edu

Specialty section:

This article was submitted to

Infectious Diseases,

a section of the journal

Frontiers in Microbiology

Received: 27 February 2020

Accepted: 24 August 2020

Published: 17 September 2020

Citation:

Vera-González N and Shukla A (2020)

Advances in Biomaterials for the

Prevention and Disruption of

Candida Biofilms.

Front. Microbiol. 11:538602.

doi: 10.3389/fmicb.2020.538602

\author{
Noel Vera-González ${ }^{1}$ and Anita Shukla ${ }^{1,2 *}$ \\ ${ }^{1}$ Center for Biomedical Engineering, School of Engineering, Brown University, Providence, Rl, United States, ${ }^{2}$ Institute for \\ Molecular and Nanoscale Innovation, Brown University, Providence, RI, United States
}

Candida species can readily colonize a multitude of indwelling devices, leading to biofilm formation. These three-dimensional, surface-associated Candida communities employ a multitude of sophisticated mechanisms to evade treatment, leading to persistent and recurrent infections with high mortality rates. Further complicating matters, the current arsenal of antifungal therapeutics that are effective against biofilms is extremely limited. Antifungal biomaterials are gaining interest as an effective strategy for combating Candida biofilm infections. In this review, we explore biomaterials developed to prevent Candida biofilm formation and those that treat existing biofilms. Surface functionalization of devices employing clinically utilized antifungals, other antifungal molecules, and antifungal polymers has been extremely effective at preventing fungi attachment, which is the first step of biofilm formation. Several mechanisms can lead to this attachment inhibition, including contact killing and release-based killing of surrounding planktonic cells. Eliminating mature biofilms is arguably much more difficult than prevention. Nanoparticles have shown the most promise in disrupting existing biofilms, with the potential to penetrate the dense fungal biofilm matrix and locally target fungal cells. We will describe recent advances in both surface functionalization and nanoparticle therapeutics for the treatment of Candida biofilms.

Keywords: Candida, biofilms, biomaterials, antifungal, surface functionalization, nanoparticles, antifungal polymers

\section{INTRODUCTION}

Candida is one of the most common causes of fungal infections worldwide, responsible for over 400,000 infections per year (Brown et al., 2012; Tsui et al., 2016). A commensal fungus that can readily become pathogenic, Candida, is known to form biofilms (Gulati and Nobile, 2016). These surface-attached, three-dimensional communities of tightly packed fungi can serve as infection strongholds, complicating treatment and leading to persistent fungemia (Li et al., 2018). Candida biofilm related infections have mortality rates as high as $41 \%$ (Rajendran et al., 2016). Biofilms protect fungal cells from the host immune system and often increase drug resistance (Mukherjee and Chandra, 2004; Nett, 2014). Biofilm fungi secrete a dense extracellular polymeric substance (EPS) that acts as a physical barrier for antifungal therapeutics, 
most of which are hydrophobic with limited ability to penetrate this matrix (Singh et al., 2018). Persister cells, which are metabolically dormant, can form as quickly as cell attachment occurs, leading to changes in gene expression, with an initial overexpression of drug efflux pumps, followed by a reduction in membrane sterol content in mature Candida biofilms (Kumamoto and Vinces, 2005; LaFleur et al., 2006). Although persister cells represent a small subpopulation within the biofilm ( $1 \%$ of all cells), their tolerance to high doses of antimicrobials allows them to readily repopulate the biofilm once the treatment has stopped, resulting in recurring infections (Galdiero et al., 2020). Quorum sensing can mediate the secretion of signaling factors affecting Candida gene expression and behavior, including filamentation (Mallick and Bennett, 2013; Tsui et al., 2016). Changes to the cell wall that enhance drug resistance can also occur; for example, cell walls that are twice as thick as planktonic cells have been observed in biofilm Candida (Nett et al., 2007; Lima et al., 2019).

The majority of biofilm-associated Candida infections arise from cells that colonize the surfaces of implanted medical devices (Coad et al., 2016). These devices range from plastic cochlear implants and subcutaneous drug delivery devices, silicone or polyurethane catheters, and acrylic dental implants, to titanium hip implants, glass-ceramics used in bone repair, metal pacemakers, and polymeric contact lenses among many others (Vargas-Blanco et al., 2017; Cavalheiro and Teixeira, 2018; Devadas et al., 2019). Treatments for these biofilm-associated infections are extremely limited, with only three primary antifungal drug classes (polyenes, azoles, and echinocandins) and a total of 21 United States Food and Drug Administration (FDA) approved antifungal drugs (Butts and Krysan, 2012; McKeny and Zito, 2020), of which only a subset have demonstrated some level of antibiofilm activity. Innovations in biomaterials have the potential to combat Candida biofilms (Figure 1).
Here, we explore recent promising approaches in this field involving surface modification with antifungal small molecules and polymers aimed at preventing biofilm formation and the design of nanoparticles aimed at both preventing and disrupting Candida biofilms.

\section{PREVENTING CANDIDA BIOFILMS USING SURFACE MODIFICATION WITH CLINICALLY UTILIZED ANTIFUNGALS}

Inhibiting Candida attachment to surfaces, the first step of biofilm formation (Figure 2: 1A), is often the most effective way to combat biofilm-associated infections. Various approaches have been investigated to prevent fungi attachment, including surface functionalization with FDA-approved antifungals using covalent and non-covalent interactions (Zelikin, 2010). Caspofungin, the only echinocandin with primary amines, is most commonly used in direct surface tethering (Coad et al., 2015; Michl et al., 2017). Caspofungin tethered titanium disks cultured with Candida albicans showed complete inhibition of fungal attachment compared to bare titanium (Figure 2: 2A,B). These same caspofungin-tethered disks implanted subcutaneously into the backs of mice and challenged with C. albicans showed $89 \%$ less Candida attached after 2 days compared to bare disks (Kucharíková et al., 2016).

In an example of non-covalent drug tethering, $\beta$-cyclodextrins (CD) were grafted to polyethylene and polypropylene surfaces (Nava-Ortiz et al., 2010), commonly used in medical devices. CDs were used to promote host-guest interactions with the hydrophobic antifungal, miconazole, while also regulating interactions with proteins and increasing hemocompatibility. These miconazole loaded CD grafted surfaces exhibited up to

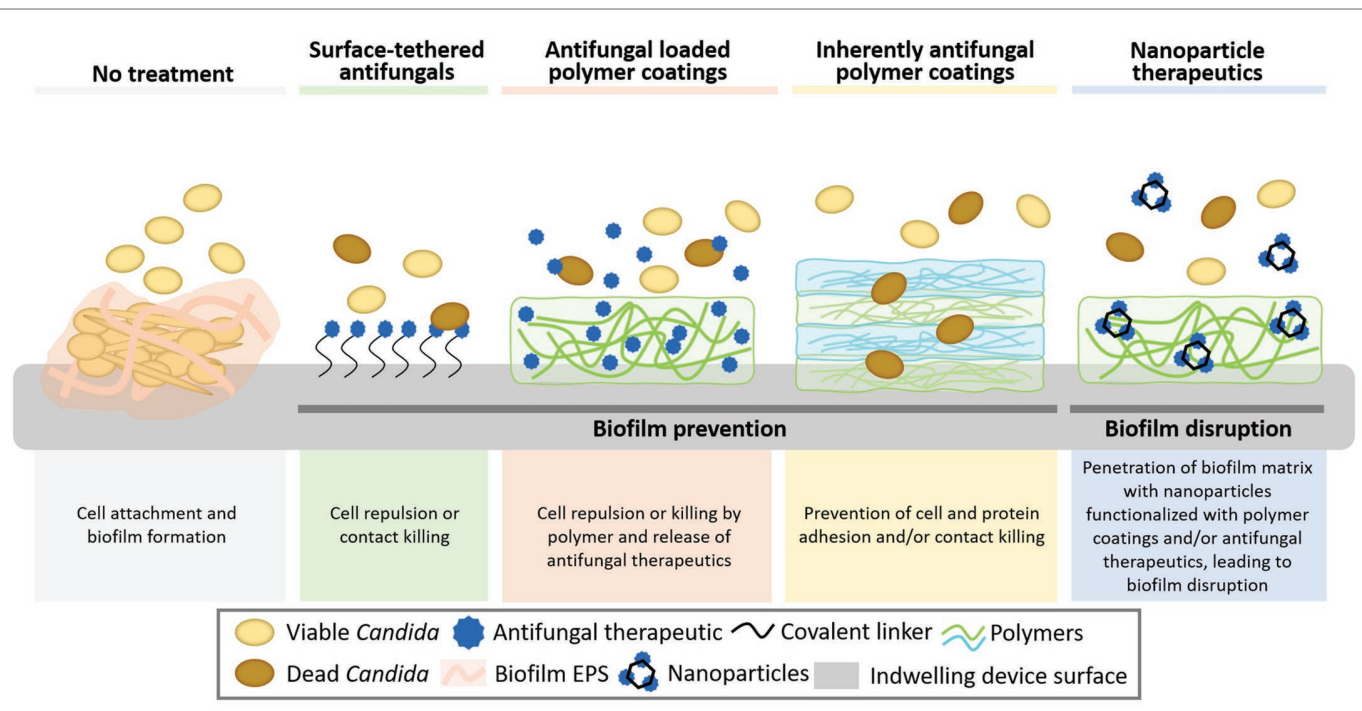

FIGURE 1 | Biomaterials strategies to combat surface-associated Candida biofilms. These strategies include direct surface functionalization with antimicrobial small molecules and natural and synthetic polymers and the use of nanoparticles, which may better penetrate the dense biofilm matrix and potentially target fungal cells. Together these strategies can prevent biofilm formation by inhibiting the initial attachment of fungi to surfaces and eradicate existing biofilms. 
1

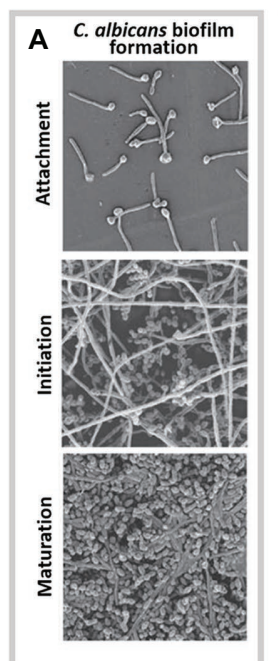

2

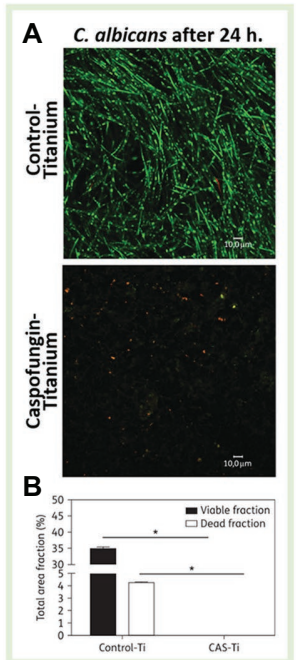

3

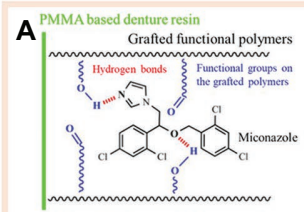

B After 28 days of drug release
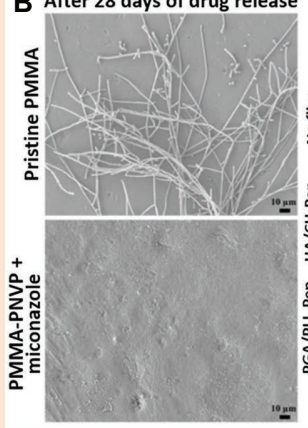

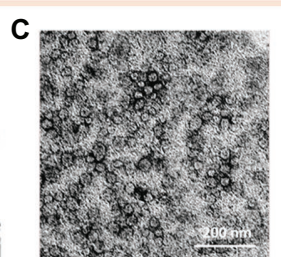

D c. albicans after 2 days

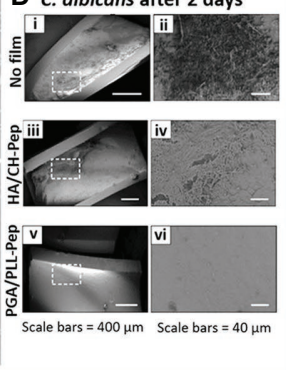

4
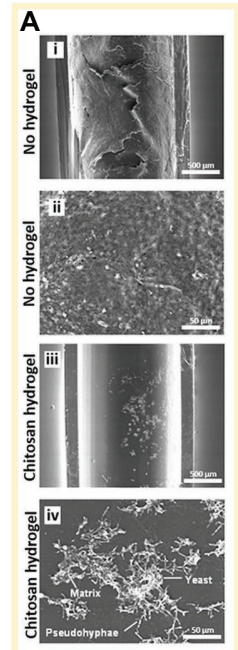

5
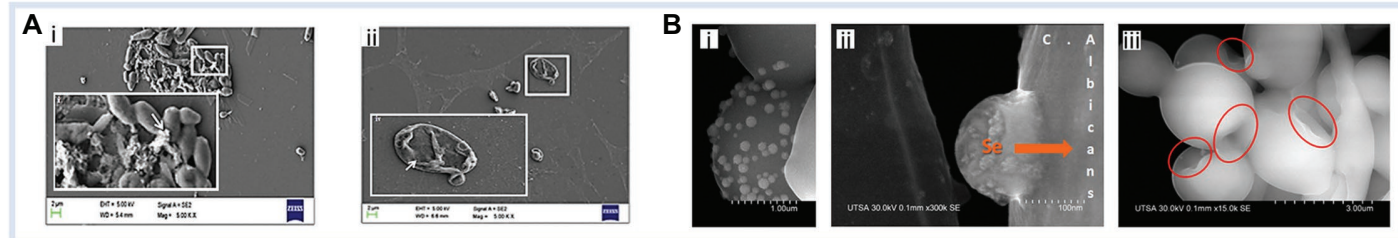

FIGURE 2 | Biomaterials for the prevention and treatment of Candida biofilms. (1) Biofilm formation of non-functionalized surfaces: (1A) Candida albicans biofilm formation (adapted with permission from Ramage et al., 2009). (2) Surface-tethered antifungals: (2A) Live/Dead staining showing caspofungin functionalized titanium disks (with caspofungin surface coverage of $\sim 2,191 \mathrm{pmol} / \mathrm{cm}^{2}$ ) inhibiting C. albicans attachment and biofilm formation compared to bare titanium (green are live cells, and red indicates membrane compromised cells; adapted with permission from Kucharíková et al., 2016). (2B) Quantification of viable cells per area in the images shown in 2A (adapted with permission from Kucharíková et al., 2016). (3) Antifungal loaded polymer coatings: (3A) Schematic illustrating miconazolepolymer hydrogen bonding (adapted with permission from Wen et al., 2016a). (3B) Miconazole loaded into poly(methyl methacrylate)-poly(1-vinyl-2-pyrrolidinone) (PMMA-PNVP) films inhibits C. albicans attachment and biofilm growth for up to 28 days compared to pristine PMMA (adapted with permission from Wen et al., 2016a). (3C) Antifungal poly(ethylene glycol) (PEG) + curcumin (CU) nanocomposites in solution after being released from graphene oxide (GO) coatings (adapted with permission from Devadas et al., 2019). (3D) Layer-by-layer (LbL) coated catheters prevent C. albicans attachment and biofilm formation after 2 days. (i) Uncoated catheters showing C. albicans attachment and biomass deposition. (ii) Magnified region outlined in 3D(i). (iii) Catheters coated with a hyaluronic acid $(\mathrm{HA}) /$ chitosan $(\mathrm{CH}) \mathrm{LbL}$ film with $\beta$-peptide showing no C. albicans attachment but some biomass deposition on the surface. (iv) Magnified region outlined in 3D(iii). (v) Poly-L-glutamic acid (PGA)/poly-L-lysine (PLL) LbL film with $\beta$-peptide coated catheters showing no cell or biomass attachment. (vi) Magnified region outlined in 3D(v) (adapted with permission from Raman et al., 2016). (4) Inherently antifungal polymer coatings: (4A) Polyurethane catheter-associated Candida parapsilosis biofilms. (i) Uncoated catheters exhibiting Candida attachment and biofilm formation. (ii) Magnified region of image 4A(i) showing a dense C. parapsilosis biofilm. (iii) Catheters coated with low molecular weight $\mathrm{CH}$ hydrogels significantly reduce Candida cell attachment and biofilm formation. (iv) Magnified region of image 4A(iii) showing biofilm disruption (adapted with permission from Silva-Dias et al., 2014). (5) Antifungal nanoparticles: (5A) Scanning electron microscopy (SEM) images of $C$. albicans biofilms on polystyrene. (i) Control biofilm cells [white arrow points to extracellular polymeric substances (EPSs)] and (ii) biofilm inhibition in the presence of ferulic acid-chitosan nanoparticles (white arrow indicates the damaged fungal cell wall; adapted with permission from Panwar et al., 2016). (5B) SEM images of selenium nanoparticles (i,ii) binding to and (iii) disrupting C. albicans cells in biofilms. The red circles indicate areas of the cell membrane, where the nanoparticles have induced shrinking and folding (adapted with permission from Guisbiers et al., 2017).

a $97 \%$ reduction in the amount of recovered C. albicans compared to a silicone control incubated with the fungus. Polymers are also commonly used to enable non-covalent functionalization with antifungals, due to their ability to form multivalent interactions promoting loading of antifungal compounds. Wen et al. grafted poly(2-hydroxyethyl methacrylate) (PHEMA) onto poly(methyl methacrylate) (PMMA) denture resins. There is great interest in preventing Candida biofilms on dental surfaces, including dentures given the prevalence of Candida in the oral microbiota; in fact, Candida is responsible for up to $67 \%$ of denture-associated stomatitis (Ramage et al., 2006). PHEMA grafting was used to load the antifungal, clotrimazole, mediated via hydrogen bonding interactions, leading to a clotrimazole surface coverage of up to $46.0 \pm 3.2 \mu \mathrm{g} / \mathrm{cm}^{2}$ compared to $5.2 \pm 0.4 \mu \mathrm{g} / \mathrm{cm}^{2}$ on bare PMMA. A sustained release of clotrimazole was observed from the PHEMA-grafted denture disks over 28 days, yielding approximately a 50 and $36 \%$ reduction in C. albicans adhesion after 1 and 28 days, respectively, compared with non PHEMA-grafted disks (Wen et al., 2016b). Grafting poly(1-vinyl-2-pyrrolidinone) (PNVP) to PMMA enabled miconazole loading of $127.0 \pm 15.1 \mu \mathrm{g} / \mathrm{cm}^{2}$, likely mediated via hydrophobic interactions and hydrogen bonding (Figure 2: 3A). PNVP-grafted resins with miconazole showed no Candida adhesion even after 28 days of drug release (Figure 2: 3B; Wen et al., 2016a). 
Along with superior biocompatibility, these functionalized materials can be used for extended biofilm prevention and have the potential to be reloaded with therapeutics.

\section{PREVENTING CANDIDA BIOFILMS USING SURFACE MODIFICATION WITH NEW ANTIFUNGAL SMALL MOLECULES AND PEPTIDES}

Although promising, surface functionalization with FDA-approved antifungals raises concerns for increased resistance to these therapeutics. Thus, there is an interest in alternative approaches to prevent Candida biofilms utilizing non-clinically used small molecules and peptides with inherent antifungal and antibiofilm properties. One example, filastatin, a potent small molecule inhibitor of Candida attachment and filamentation was recently identified in a screen of 30,000 compounds (Fazly et al., 2013). Vargas-Blanco et al. (2017) found that incubation of C. albicans with various biomaterials in the presence of filastatin can inhibit Candida attachment to these materials. Adsorption of filastatin on dental resin and silicone showed that Candida cell attachment was reduced on these materials by 62.7 and $79.7 \%$, respectively, compared to uncoated controls. By incorporating filastatin into the silicone matrix during polymerization a 6.5 -fold reduction in C. albicans adhesion compared to untreated silicone controls was observed (Vargas-Blanco et al., 2017). Other small molecule biofilm inhibitors specifically interrupt Candida quorum sensing. These molecules include furanones, which are plant synthesized compounds that prevent microbial fouling on the plant surface. Devadas et al. (2019) coated common catheter materials with a furanone embedded polycaprolactone matrix. These polymer coatings retained $85 \%$ or more of the total loaded furanone over at least 30 days in solution. The attachment of clinical isolates of Candida tropicalis, Candida glabrata, and Candida krusei on these coated catheters was completely inhibited as determined by scanning electron microscopy (SEM; Devadas et al., 2019). Other plant derived compounds have also shown activity against Candida biofilms when combined with biomaterials. Recently, clove oil and red thyme oil incorporated in polycaprolactone electrospun nanofibers led to a 60 and $80 \%$ reduction in C. tropicalis attachment, respectively (Sahal et al., 2019). Initial results with these small molecules are promising; future studies will likely examine functionalization via covalent tethering or affinity-based interactions with these compounds to enable long-term antibiofilm activity.

Combination approaches to prevent Candida biofilms involving the inhibition of fungal cell attachment and simultaneous killing of planktonic fungi have also been investigated. Palmieri et al. (2018) developed a multilayered coating by drop-casting graphene oxide (GO) on polyurethane catheters, followed by curcumin (CU) and poly(ethylene glycol) (PEG). The GO was included to prevent $C$. albicans attachment due to its ability to generate oxidative stress and physically disrupt the cell wall and membrane. $\mathrm{CU}+$ PEG self-assembled nanocomposites (75-125 nm in diameter; Figure 2: 3C) were released from these coatings inhibiting planktonic $C$. albicans growth, with a minimum inhibitory concentration of $10.6 \mu \mathrm{g} / \mathrm{ml}$. The complete catheter coating inhibited C. albicans attachment in vitro after $24 \mathrm{~h}$ with less than $20 \%$ biofilm formation compared to uncoated controls (Palmieri et al., 2018).

As an alternative to solvent casting or vapor deposition approaches, many biomedical surfaces have been coated via layer-by-layer (LbL) self-assembly to develop antifungal coatings. LbL assembly is a multilayer film fabrication method that involves alternating the adsorption of molecules and macromolecules (e.g., polyelectrolytes, peptides, proteins, small molecules, etc.) with complementary functionalities most commonly by dip coating (Shukla and Almeida, 2014; Alkekhia and Shukla, 2019; Alkekhia et al., 2020). LbL films have been combined with antifungal peptides to exhibit remarkable antibiofilm properties. Antifungal peptides are considered potent and broad-spectrum antifungals; due to their multiple mechanisms of action, fungi are often unable to develop resistance to these peptides (Oshiro et al., 2019). These peptides are most commonly amphiphilic and cationic allowing them to readily interact with the fungal cell membrane, causing cell death (Karlsson et al., 2010). Raman et al. (2016) assembled an LbL film with hyaluronic acid (HA) and chitosan $(\mathrm{CH})$ on catheter surfaces and used it as a reservoir for a synthetic antifungal $\beta$-peptide. The luminal surface of polyurethane catheters coated with these LbL films without any $\beta$-peptide was able to reduce viable $C$. albicans by approximately 25 -fold after $6 \mathrm{~h}$ of exposure when compared to uncoated polyurethane catheters, demonstrating the innate antifungal properties of this polymeric coating. When passively loaded with the antifungal $\beta$-peptide, sustained release of the $\beta$-peptide was achieved over 50 days with complete eradication of planktonic $C$. albicans in vitro. Catheters coated with the $\beta$-peptide-loaded films tested in a rat central venous catheter model exhibited almost no fungal cells following 2 days [Figure 2: $3 \mathbf{D}(\mathbf{i}-\mathbf{i v})]$. However, this coated surface was found to contain a network of host proteins, which can yield complications, including fouling with red blood cells, which can stimulate platelet production. Another film architecture examined in the same study utilizing $\beta$-peptideloaded poly-L-lysine (PLL) and poly-L-glutamic acid (PGA) LbL films exhibited both a complete lack of Candida cell attachment and host proteins when tested in the same in vivo model [Figure 2: $3 \mathbf{D}(\mathbf{v}, \mathbf{v i})$ ], emphasizing the importance of polymer choice in preventing overall fouling (Raman et al., 2016). PMMA denture disks were also recently coated with an LbL film containing the cationic mammalian salivary antifungal peptide, histatin-5 (H-5), and HA with a final H-5 layer. SEM images confirmed over 4 weeks that these LbL coated surfaces were able to completely prevent Candida attachment (Wen et al., 2018).

Many other small molecules and peptides not yet incorporated into biomaterials have demonstrated antibiofilm activity. Among these are newly synthesized imidazole derivatives, which have been found to prevent Candida biofilm formation and disrupt existing biofilms (Ribeiro et al., 2014; Gabriel et al., 2019). Thiazolylhydrazone derivatives have also recently emerged as effective antifungal compounds with low mammalian cell toxicity (Cruz et al., 2018). 
2,6-Bis[(E)-(4-pyridyl)methylidene]cyclohexanone, an antiparasitic compound, was also found to exhibit antifungal properties including the inhibition of Candida filamentation, crucial in biofilm formation (de Sá et al., 2018). Antifungal peptide derivatives of H-5 are also being explored (Sultan et al., 2019), and other host defense peptides such as innate defense regulator 1018 and porcine cathelicidins have recently been shown to possess antifungal and antibiofilm properties against Candida (Lyu et al., 2016; Freitas et al., 2017). These compounds are potential candidates for incorporation into antifungal biomaterials.

\section{PREVENTING CANDIDA BIOFILMS USING POLYMER-ONLY COATINGS}

Many polymers themselves possess inherent antifouling, antifungal, and/or antibiofilm properties, while being less susceptible to resistance compared with small molecule antifungals; therefore, the use of polymer-only coatings for combating Candida biofilms has gained significant interest. For example, chitosan, a naturally derived polysaccharide, has been widely incorporated into hydrogels and coatings to prevent Candida attachment and biofilm formation (Carlson et al., 2008; Ailincai et al., 2016; Tan et al., 2016). It is hypothesized that chitosan interacts electrostatically via its positively charged amino groups with anionic moieties on microbial species leading to increased membrane permeability and eventual cell death (Jung et al., 2020). In a recent study, polyurethane intravenous catheters were coated with low molecular weight $(50 \mathrm{kDa})$ chitosan hydrogels, implanted subcutaneously into mice, and subsequently challenged with Candida parapsilosis. Following 7 days, the chitosan-coated catheters reduced Candida metabolic activity by $\sim 96 \%$ when compared to uncoated catheters, showcasing the ability of polymer-only coatings free of small molecule antifungals to achieve excellent antibiofilm activity. Reduced biomass on these chitosan coated catheters was shown using SEM (Figure 2: 4A; Silva-Dias et al., 2014). Chitosan has also been modified to enhance its antibiofilm properties. Jung et al. (2019) examined the use of amphiphilic quaternary ammonium chitosans (AQACs) in LbL coatings. LbL films containing sodium alginate and AQAC, effectively prevented cell attachment on coated PMMA substrates (Jung et al., 2019). AQACs have been shown to disrupt mature Candida biofilms by interacting electrostatically with the negatively charged biofilm surface (Jung et al., 2020). Coatings with other polymers including imidazolium salt (IS) conjugated poly(L-lactide) (PLA) have also been used to effectively prevent Candida attachment on coated surfaces (Schrekker et al., 2016).

\section{NANOPARTICLES FOR THE PREVENTION OF FUNGAL CELL ATTACHMENT AND BIOFILM ERADICATION}

Despite the progress that has been made in antifungal surface functionalization, these approaches are limited in their ability to treat mature biofilms. Nanoparticles are a promising strategy to eradicate existing biofilms, with the potential to carry, stabilize, and protect therapeutic payloads, penetrate the EPS, target fungal cells, and be internalized (Ikuma et al., 2015; Qayyum and Khan, 2016; Stone et al., 2016). Several strategies have been used to develop nanoparticles for the treatment of fungal infections, from using inorganic compounds to antimicrobial polymers (Ahmad et al., 2016; Amaral et al., 2019). In an example of the latter approach, chitosan nanoparticles (20-30 nm diameter) were recently examined for their ability to inhibit C. albicans biofilm growth, following initial cell attachment. Incubation with chitosan nanoparticles for $3 \mathrm{~h}$ led to a greater than $50 \%$ reduction in biofilm mass compared to non-treated controls (Ikono et al., 2019). While these chitosan nanoparticles exhibited some inherent antibiofilm activity, they were unable to entirely inhibit or disrupt Candida biofilms. Panwar et al. (2016) instead incorporated ferulic acid, a plant derived small molecule with known antibiofilm properties (Teodoro et al., 2015), into chitosan nanoparticles ( 115 nm diameter). On its own, ferulic acid cannot efficiently penetrate fungal biofilms; however, when incorporated into chitosan nanoparticles and incubated with C. albicans biofilms, a significant reduction in fungal metabolic activity was observed $(22.5 \%$ normalized to an untreated biofilm following $24 \mathrm{~h}$ ). While the mechanism of these nanoparticles is not fully understood, it is believed that their strong cationic surface charge allows them to localize to and disrupt the fungal cell membrane while the surface bound ferulic acid interrupts Candida oxidative phosphorylation. This cell damage is evident in SEM images [Figure 2: 5A(ii)] when compared to healthy biofilm cells [Figure 2: 5A(i); Panwar et al., 2016].

Lipid-based self-assembled nanoparticles have also shown promise in penetrating the biofilm matrix and in targeting fungal cells. AmBisome ${ }^{\circledR}$, a widely utilized liposomal formulation of amphotericin B, is able to disrupt Candida biofilms while free amphotericin B is unable to do this (Stone et al., 2016). AmBisome has a strong affinity for Candida cells, electrostatically interacting with the cell wall before binding to the cell membrane at sites of high ergosterol content (Soo Hoo, 2017), which may promote their activity against biofilm Candida cells, which have been shown to have thicker cell walls (Nett et al., 2007). Liposomal amphotericin B has also been immobilized on biomaterial surfaces for the prevention of biofilm formation (Alves et al., 2019). In our recent work, we have shown that liposomes encapsulating anidulafungin, the latest echinocandin approved by the FDA, are effective against mature C. albicans biofilms, reducing metabolic activity to approximately $46 \%$ compared to untreated controls over $24 \mathrm{~h}$. Biofilms treated with an equivalent concentration of free anidulafungin did not reduce metabolic activity, further emphasizing the importance of nanoformulations in the treatment of Candida biofilms (Vera-González et al., 2020).

In addition to organic nanoparticles, inorganic nanoparticles have also been widely utilized for their antimicrobial properties, most commonly including silver and silica nanoparticles (Cousins et al., 2007; Monteiro et al., 2011; Silva et al., 2013). Silver nanoparticles were recently shown to inhibit biofilm formation 
of multi-drug resistant Candida auris (Lara et al., 2020), an emerging fungal threat with the unique ability to survive on surfaces for several weeks (Welsh et al., 2017). Selenium nanoparticles, which are less toxic to mammalian cells than silver nanoparticles, have only recently been explored for their antimicrobial properties (Huang et al., 2016). Guisbiers et al. (2017) demonstrated that $\sim 100 \mathrm{~nm}$ selenium nanoparticles successfully inhibited the formation of C. albicans biofilms by attaching to and penetrating through the cell wall (Figure 2: 5B), replacing sulfur with selenium in important biochemical processes. These particles were able to reduce fungal burden in mature biofilms by over $50 \%$ at a nanoparticle concentration of as low as 26 ppm (Guisbiers et al., 2017). Inorganic nanoparticles have also been combined with antimicrobial therapeutics, to enhance antifungal properties. de Alteriis et al. (2018) conjugated the mammalian antimicrobial cathelicidin peptide, indolicidin, to the surface of gold nanoparticles $(5 \mathrm{~nm}$ diameter) in order to protect it from proteolytic degradation and self-aggregation. These particles were able to penetrate and disrupt mature biofilms, eradicating over $50 \%$ of the cells for the most C. albicans and C. tropicalis strains tested after $24 \mathrm{~h}$ of treatment when compared to untreated biofilms, with a hypothesized mechanism involving penetration of the fungal cell membrane and inhibition of intracellular targets, arresting cell metabolism (de Alteriis et al., 2018).

\section{CONCLUSIONS AND PERSPECTIVES}

We have discussed several biomaterials strategies from surface functionalization to nanoparticle drug delivery for the prevention and disruption of Candida biofilms. Other approaches that can be combined with biomaterials to functionalize surfaces prone to the biofilm formation in the near future include the use of enzymes that target and digest EPS components (Nett, 2014), identification of new drug targets, including inhibition of Candida extracellular vesicles (Zarnowski et al., 2018), and incorporation

\section{REFERENCES}

Ahmad, A., Wei, Y., Syed, F., Tahir, K., Taj, R., Khan, A. U., et al. (2016). Amphotericin B-conjugated biogenic silver nanoparticles as an innovative strategy for fungal infections. Microb. Pathog. 99, 271-281. doi: 10.1016/j.micpath.2016.08.031

Ailincai, D., Marin, L., Morariu, S., Mares, M., Bostanaru, A. -C., Pinteala, M., et al. (2016). Dual crosslinked iminoboronate-chitosan hydrogels with strong antifungal activity against Candida planktonic yeasts and biofilms. Carbohydr. Polym. 152, 306-316. doi: 10.1016/j.carbpol.2016.07.007

Alkekhia, D., Hammond, P. T., and Shukla, A. (2020). Layer-by-layer biomaterials for drug delivery. Annu. Rev. Biomed. Eng. 22, 1-24. doi: 10.1146/annurevbioeng-060418-052350

Alkekhia, D., and Shukla, A. (2019). Influence of poly-L-lysine molecular weight on antibacterial efficacy in polymer multilayer films. J. Biomed. Mater. Res. A 107, 1324-1339. doi: 10.1002/jbm.a.36645

Alves, D., Vaz, A. T., Grainha, T., Rodrigues, C. F., and Pereira, M. O. (2019). Design of an antifungal surface embedding liposomal amphotericin b through a mussel adhesive-inspired coating strategy. Front. Chem. 7:431. doi: 10.3389/ fchem.2019.00431

Amaral, A. C., Saavedra, P. H. V., Oliveira Souza, A. C., de Melo, M. T., Tedesco, A. C., Morais, P. C., et al. (2019). Miconazole loaded chitosan-based of polymers, such as nylon-3 that have potent and selective activity against Candida biofilms (Liu et al., 2014, 2015).

While many advances have been made, development of antifungal biomaterials lags behind the development of antibacterial materials. There is a need for expansion and innovation in antifungal biomaterials, and an emphasis must be placed on advancing technologies beyond preclinical testing. Attention must also be given to polymicrobial biofilms, comprised of multiple fungal and bacterial species, which are currently understudied (Harriott and Noverr, 2009). It is estimated that more than $50 \%$ of C. albicans infections are polymicrobial in nature (Harriott and Noverr, 2011; Nash et al., 2016). Undoubtedly, it will be critical to use multi-pronged strategies combining effective biomaterials approaches (e.g., surface coatings with nanoparticles) to successfully combat Candida and other microbial biofilms.

\section{AUTHOR CONTRIBUTIONS}

NV-G and AS organized, prepared, and approved the final version of this manuscript. All authors contributed to the article and approved the submitted version.

\section{FUNDING}

AS and NV-G acknowledge support from the Office of Naval Research (grant N000141712651) awarded to AS and from a National Science Foundation Graduate Research Fellowship awarded to NV-G (grant 1644760).

\section{ACKNOWLEDGMENTS}

AS and NV-G thank Brown University graduate student, Carly Deusenbery, and postdoctoral researcher, Dr. Akram Abbasi, for useful discussions related to figure preparation. nanoparticles for local treatment of vulvovaginal candidiasis fungal infections. Colloids Surf. B 174, 409-415. doi: 10.1016/j.colsurfb.2018.11.048

Brown, G. D., Denning, D. W., Gow, N. A. R., Levitz, S. M., Netea, M. G., and White, T. C. (2012). Hidden killers: human fungal infections. Sci. Transl. Med. 4:165rv13. doi: 10.1126/scitranslmed.3004404

Butts, A., and Krysan, D. J. (2012). Antifungal drug discovery: something old and something new. PLoS Pathog. 8:e1002870. doi: 10.1371/journal.ppat.1002870

Carlson, R. P., Taffs, R., Davison, W. M., and Stewart, P. S. (2008). Anti-biofilm properties of chitosan-coated surfaces. J. Biomater. Sci. Polym. Ed. 19, 1035-1046. doi: 10.1163/156856208784909372

Cavalheiro, M., and Teixeira, M. C. (2018). Candida biofilms: threats, challenges, and promising strategies. Front. Med. 5:28. doi: 10.3389/fmed.2018.00028

Coad, B. R., Griesser, H. J., Peleg, A. Y., and Traven, A. (2016). Anti-infective surface coatings: design and therapeutic promise against device-associated infections. PLoS Pathog. 12:e1005598. doi: 10.1371/journal.ppat.1005598

Coad, B. R., Lamont-Friedrich, S. J., Gwynne, L., Jasieniak, M., Griesser, S. S., Traven, A., et al. (2015). Surface coatings with covalently attached caspofungin are effective in eliminating fungal pathogens. J. Mater. Chem. B 3, 8469-8476. doi: 10.1039/C5TB00961H

Cousins, B. G., Allison, H. E., Doherty, P. J., Edwards, C., Garvey, M. J., Martin, D. S., et al. (2007). Effects of a nanoparticulate silica substrate on 
cell attachment of Candida albicans. J. Appl. Microbiol. 102, 757-765. doi: 10.1111/j.1365-2672.2006.03124.x

Cruz, L., Lopes, L., de Camargo Ribeiro, F., de Sá, N., Lino, C., Tharmalingam, N., et al. (2018). Anti-Candida albicans activity of thiazolylhydrazone derivatives in invertebrate and murine models. J. Fungi 4:134. doi: 10.3390/jof4040134

de Alteriis, E., Maselli, V., Falanga, A., Galdiero, S., Di Lella, F. M., Gesuele, R., et al. (2018). Efficiency of gold nanoparticles coated with the antimicrobial peptide indolicidin against biofilm formation and development of Candida spp. clinical isolates. Infect. Drug Resist. 11, 915-925. doi: 10.2147/IDR.S164262

de Sá, N. P., de Paula, L. F. J., Lopes, L. F. F., Cruz, L. I. B., Matos, T. T. S., Lino, C. I., et al. (2018). In vivo and in vitro activity of a bis-arylidenecycloalkanone against fluconazole-susceptible and -resistant isolates of Candida albicans. J. Glob. Antimicrob. Resist. 14, 287-293. doi: 10.1016/j.jgar.2018.04.012

Devadas, S. M., Nayak, U. Y., Narayan, R., Hande, M. H., and Ballal, M. (2019). 2,5-Dimethyl-4-hydroxy-3(2H)-furanone as an anti-biofilm agent against non-Candida albicans Candida species. Mycopathologia 184, 403-411. doi: 10.1007/s11046-019-00341-y

Fazly, A., Jain, C., Dehner, A. C., Issi, L., Lilly, E. A., Ali, A., et al. (2013). Chemical screening identifies filastatin, a small molecule inhibitor of Candida albicans adhesion, morphogenesis, and pathogenesis. Proc. Natl. Acad. Sci. U. S. A. 110, 13594-13599. doi: 10.1073/pnas.1305982110

Freitas, C. G., Lima, S. M. F., Freire, M. S., Cantuária, A. P. C., Júnior, N. G. O., Santos, T. S., et al. (2017). An immunomodulatory peptide confers protection in an experimental candidemia murine model. Antimicrob. Agents Chemother. 61, e02518-e02616. doi: 10.1128/AAC.02518-16

Gabriel, C., Grenho, L., Cerqueira, F., Medeiros, R., Dias, A. M., Ribeiro, A. I., et al. (2019). Inhibitory effect of 5-aminoimidazole-4-carbohydrazonamides derivatives against Candida spp. biofilm on nanohydroxyapatite substrate. Mycopathologia 184, 775-786. doi: 10.1007/s11046-019-00400-4

Galdiero, E., de Alteriis, E., De Natale, A., D’Alterio, A., Siciliano, A., Guida, M., et al. (2020). Eradication of Candida albicans persister cell biofilm by the membranotropic peptide gH625. Sci. Rep. 10:5780. doi: 10.1038/s41598-020-62746-w

Guisbiers, G., Lara, H. H., Mendoza-Cruz, R., Naranjo, G., Vincent, B. A., Peralta, X. G., et al. (2017). Inhibition of Candida albicans biofilm by pure selenium nanoparticles synthesized by pulsed laser ablation in liquids. Nanomedicine 13, 1095-1103. doi: 10.1016/J.NANO.2016.10.011

Gulati, M., and Nobile, C. J. (2016). Candida albicans biofilms: development, regulation, and molecular mechanisms. Microbes Infect. 18, 310-321. doi: 10.1016/j.micinf.2016.01.002

Harriott, M. M., and Noverr, M. C. (2009). Candida albicans and Staphylococcus aureus form polymicrobial biofilms: effects on antimicrobial resistance. Antimicrob. Agents Chemother. 53, 3914-3922. doi: 10.1128/AAC.00657-09

Harriott, M. M., and Noverr, M. C. (2011). Importance of Candida-bacterial polymicrobial biofilms in disease. Trends Microbiol. 19, 557-563. doi: 10.1016/j. tim.2011.07.004

Huang, X., Chen, X., Chen, Q., Yu, Q., Sun, D., and Liu, J. (2016). Investigation of functional selenium nanoparticles as potent antimicrobial agents against superbugs. Acta Biomater. 30, 397-407. doi: 10.1016/j.actbio.2015.10.041

Ikono, R., Vibriani, A., Wibowo, I., Saputro, K. E., Muliawan, W., Bachtiar, B. M., et al. (2019). Nanochitosan antimicrobial activity against Streptococcus mutans and Candida albicans dual-species biofilms. BMC Res. Notes 12:383. doi: 10.1186/s13104-019-4422-x

Ikuma, K., Decho, A. W., and Lau, B. L. T. (2015). When nanoparticles meet biofilms-interactions guiding the environmental fate and accumulation of nanoparticles. Front. Microbiol. 6:591. doi: 10.3389/fmicb.2015.00591

Jung, J., Bae, Y., Kwan Cho, Y., Ren, X., and Sun, Y. (2020). Structural insights into conformation of amphiphilic quaternary ammonium chitosans to control fungicidal and anti-biofilm functions. Carbohydr. Polym. 228:115391. doi: 10.1016/j.carbpol.2019.115391

Jung, J., Li, L., Yeh, C. -K., Ren, X., and Sun, Y. (2019). Amphiphilic quaternary ammonium chitosan/sodium alginate multilayer coatings kill fungal cells and inhibit fungal biofilm on dental biomaterials. Mater. Sci. Eng. C 104:109961. doi: 10.1016/j.msec.2019.109961

Karlsson, A. J., Flessner, R. M., Gellman, S. H., Lynn, D. M., and Palecek, S. P. (2010). Polyelectrolyte multilayers fabricated from antifungal $\beta$-peptides: design of surfaces that exhibit antifungal activity against Candida albicans. Biomacromolecules 11, 2321-2328. doi: 10.1021/bm100424s

Kucharíková, S., Gerits, E., De Brucker, K., Braem, A., Ceh, K., Majdič, G., et al. (2016). Covalent immobilization of antimicrobial agents on titanium prevents Staphylococcus aureus and Candida albicans colonization and biofilm formation. J. Antimicrob. Chemother. 71, 936-945. doi: 10.1093/jac/dkv437

Kumamoto, C. A., and Vinces, M. D. (2005). Alternative Candida albicans lifestyles: growth on surfaces. Annu. Rev. Microbiol. 59, 113-133. doi: 10.1146/ annurev.micro.59.030804.121034

LaFleur, M. D., Kumamoto, C. A., and Lewis, K. (2006). Candida albicans biofilms produce antifungal-tolerant persister cells. Antimicrob. Agents Chemother. 50, 3839-3846. doi: 10.1128/AAC.00684-06

Lara, H. H., Ixtepan-Turrent, L., Jose Yacaman, M., and Lopez-Ribot, J. (2020). Inhibition of Candida auris biofilm formation on medical and environmental surfaces by silver nanoparticles. ACS Appl. Mater. Interfaces 12, 21183-21191. doi: $10.1021 /$ acsami.9b20708

Li, W. -S., Chen, Y. -C., Kuo, S. -F., Chen, F. -J., and Lee, C. -H. (2018). The impact of biofilm formation on the persistence of candidemia. Front. Microbiol. 9:1196. doi: 10.3389/fmicb.2018.01196

Lima, S. L., Colombo, A. L., and de Almeida Junior, J. N. (2019). Fungal cell wall: emerging antifungals and drug resistance. Front. Microbiol. 10:2573. doi: $10.3389 /$ fmicb.2019.02573

Liu, R., Chen, X., Falk, S. P., Masters, K. S., Weisblum, B., and Gellman, S. H. (2015). Nylon-3 polymers active against drug-resistant Candida albicans biofilms. J. Am. Chem. Soc. 137, 2183-2186. doi: 10.1021/ja512567y

Liu, R., Chen, X., Falk, S. P., Mowery, B. P., Karlsson, A. J., Weisblum, B., et al. (2014). Structure-activity relationships among antifungal nylon-3 polymers: identification of materials active against drug-resistant strains of Candida albicans. J. Am. Chem. Soc. 136, 4333-4342. doi: 10.1021/ ja500036r

Lyu, Y., Yang, Y., Lyu, X., Dong, N., and Shan, A. (2016). Antimicrobial activity, improved cell selectivity and mode of action of short PMAP-36-derived peptides against bacteria and Candida. Sci. Rep. 6:27258. doi: 10.1038/ srep27258

Mallick, E. M., and Bennett, R. J. (2013). Sensing of the microbial neighborhood by Candida albicans. PLoS Pathog. 9:e1003661. doi: 10.1371/journal.ppat.1003661

McKeny, P. T., and Zito, P. M. (2020). Antifungal antibiotics. StatPearls publishing. Available at: http://www.ncbi.nlm.nih.gov/pubmed/30844195 (Accessed February 6, 2020).

Michl, T. D., Giles, C., Mocny, P., Futrega, K., Doran, M. R., Klok, H. -A., et al. (2017). Caspofungin on ARGET-ATRP grafted PHEMA polymers: enhancement and selectivity of prevention of attachment of Candida albicans. Biointerphases 12:05G602. doi: 10.1116/1.4986054

Monteiro, D. R., Gorup, L. F., Silva, S., Negri, M., de Camargo, E. R., Oliveira, R., et al. (2011). Silver colloidal nanoparticles: antifungal effect against adhered cells and biofilms of Candida albicans and Candida glabrata. Biofouling 27, 711-719. doi: 10.1080/08927014.2011.599101

Mukherjee, P., and Chandra, J. (2004). Candida biofilm resistance. Drug Resist. Updat. 7, 301-309. doi: 10.1016/j.drup.2004.09.002

Nash, E. E., Peters, B. M., Fidel, P. L., and Noverr, M. C. (2016). Morphologyindependent virulence of Candida species during polymicrobial intra-abdominal infections with Staphylococcus aureus. Infect. Immun. 84, 90-98. doi: 10.1128/ IAI.01059-15

Nava-Ortiz, C. A. B., Burillo, G., Concheiro, A., Bucio, E., Matthijs, N., Nelis, H., et al. (2010). Cyclodextrin-functionalized biomaterials loaded with miconazole prevent Candida albicans biofilm formation in vitro. Acta Biomater. 6, 1398-1404. doi: 10.1016/j.actbio.2009.10.039

Nett, J. E. (2014). Future directions for anti-biofilm therapeutics targeting Candida. Expert Rev. Anti-Infect. Ther. 12, 375-382. doi: 10.1586/ 14787210.2014 .885838

Nett, J., Lincoln, L., Marchillo, K., Massey, R., Holoyda, K., Hoff, B., et al. (2007). Putative role of $\beta-1,3$ glucans in Candida albicans biofilm resistance. Antimicrob. Agents Chemother. 51, 510-520. doi: 10.1128/AAC.01056-06

Oshiro, K. G. N., Rodrigues, G., Monges, B. E. D., Cardoso, M. H., and Franco, O. L. (2019). Bioactive peptides against fungal biofilms. Front. Microbiol. 10:2169. doi: 10.3389/fmicb.2019.02169

Palmieri, V., Bugli, F., Cacaci, M., Perini, G., De Maio, F., Delogu, G., et al. (2018). Graphene oxide coatings prevent Candida albicans biofilm formation with a controlled release of curcumin-loaded nanocomposites. Nanomedicine 13, 2867-2879. doi: 10.2217/nnm-2018-0183

Panwar, R., Pemmaraju, S. C., Sharma, A. K., and Pruthi, V. (2016). Efficacy of ferulic acid encapsulated chitosan nanoparticles against Candida albicans biofilm. Microb. Pathog. 95, 21-31. doi: 10.1016/j.micpath.2016.02.007 
Qayyum, S., and Khan, A. U. (2016). Nanoparticles vs. biofilms: a battle against another paradigm of antibiotic resistance. Med. Chem. Commun. 7, 1479-1498. doi: 10.1039/C6MD00124F

Rajendran, R., Sherry, L., Nile, C. J., Sherriff, A., Johnson, E. M., Hanson, M. F., et al. (2016). Biofilm formation is a risk factor for mortality in patients with Candida albicans bloodstream infection-Scotland, 2012-2013. Clin. Microbiol. Infect. 22, 87-93. doi: 10.1016/j.cmi.2015.09.018

Ramage, G., Martínez, J. P., and López-Ribot, J. L. (2006). Candida biofilms on implanted biomaterials: a clinically significant problem. FEMS Yeast Res. 6, 979-986. doi: 10.1111/j.1567-1364.2006.00117.x

Ramage, G., Mowat, E., Jones, B., Williams, C., and Lopez-Ribot, J. (2009). Our current understanding of fungal biofilms. Crit. Rev. Microbiol. 35, 340-355. doi: 10.3109/10408410903241436

Raman, N., Marchillo, K., Lee, M. -R., de L Rodríguez López, A., Andes, D. R., Palecek, S. P., et al. (2016). Intraluminal release of an antifungal $\beta$-peptide enhances the antifungal and anti-biofilm activities of multilayer-coated catheters in a rat model of venous catheter infection. ACS Biomater. Sci. Eng. 2, 112-121. doi: 10.1021/acsbiomaterials.5b00427

Ribeiro, A. I., Gabriel, C., Cerqueira, F., Maia, M., Pinto, E., Sousa, J. C., et al. (2014). Synthesis and antimicrobial activity of novel 5-aminoimidazole-4carboxamidrazones. Bioorg. Med. Chem. Lett. 24, 4699-4702. doi: 10.1016/j. bmcl.2014.08.025

Sahal, G., Nasseri, B., Ebrahimi, A., and Bilkay, I. S. (2019). Electrospun essential oil-polycaprolactone nanofibers as antibiofilm surfaces against clinical Candida tropicalis isolates. Biotechnol. Lett. 41, 511-522. doi: 10.1007/s10529-019-02660-y

Schrekker, C. M. L., Sokolovicz, Y. C. A., Raucci, M. G., Selukar, B. S., Klitzke, J. S., Lopes, W., et al. (2016). Multitask imidazolium salt additives for innovative poly(L-lactide) biomaterials: morphology control, Candida spp. biofilm inhibition, human mesenchymal stem cell biocompatibility, and skin tolerance. ACS Appl. Mater. Interfaces 8, 21163-21176. doi: 10.1021/acsami.6b06005

Shukla, A., and Almeida, B. (2014). Advances in cellular and tissue engineering using layer-by-layer assembly. Wiley Interdiscip. Rev. Nanomed. Nanobiotechnol. 6, 411-421. doi: 10.1002/wnan.1269

Silva, S., Pires, P., Monteiro, D. R., Negri, M., Gorup, L. F., Camargo, E. R., et al. (2013). The effect of silver nanoparticles and nystatin on mixed biofilms of Candida glabrata and Candida albicans on acrylic. Med. Mycol. 51, 178-184. doi: 10.3109/13693786.2012.700492

Silva-Dias, A., Palmeira-de-Oliveira, A., Miranda, I. M., Branco, J., Cobrado, L., Monteiro-Soares, M., et al. (2014). Anti-biofilm activity of low-molecular weight chitosan hydrogel against Candida species. Med. Microbiol. Immunol. 203, 25-33. doi: 10.1007/s00430-013-0311-4

Singh, R., Kumari, A., Kaur, K., Sethi, P., and Chakrabarti, A. (2018). Relevance of antifungal penetration in biofilm-associated resistance of Candida albicans and non-albicans Candida species. J. Med. Microbiol. 67, 922-926. doi: $10.1099 / \mathrm{jmm} .0 .000757$

Soo Hoo, L. (2017). Fungal fatal attraction: a mechanistic review on targeting liposomal amphotericin B (AmBisome $\left.{ }^{\circledR}\right)$ to the fungal membrane. J. Liposome Res. 27, 180-185. doi: 10.1080/08982104.2017.1360345

Stone, N. R. H., Bicanic, T., Salim, R., and Hope, W. (2016). Liposomal amphotericin B $(\text { AmBisome })^{\circ}$ : a review of the pharmacokinetics, pharmacodynamics, clinical experience and future directions. Drugs 76, 485-500. doi: 10.1007/s40265-016-0538-7
Sultan, A. S., Vila, T., Hefni, E., Karlsson, A. J., and Jabra-Rizk, M. A. (2019). Evaluation of the antifungal and wound-healing properties of a novel peptidebased bioadhesive hydrogel formulation. Antimicrob. Agents Chemother. 63, e00888-e00919. doi: 10.1128/AAC.00888-19

Tan, Y., Leonhard, M., Moser, D., Ma, S., and Schneider-Stickler, B. (2016). Inhibition of mixed fungal and bacterial biofilms on silicone by carboxymethyl chitosan. Colloids Surf. B 148, 193-199. doi: 10.1016/j.colsurfb.2016.08.061

Teodoro, G. R., Ellepola, K., Seneviratne, C. J., and Koga-Ito, C. Y. (2015). Potential use of phenolic acids as anti-Candida agents: a review. Front. Microbiol. 6:1420. doi: $10.3389 /$ fmicb.2015.01420

Tsui, C., Kong, E. F., and Jabra-Rizk, M. A. (2016). Pathogenesis of Candida albicans biofilm. Pathog. Dis. 74:ftw018. doi: 10.1093/femspd/ftw018

Vargas-Blanco, D., Lynn, A., Rosch, J., Noreldin, R., Salerni, A., Lambert, C. et al. (2017). A pre-therapeutic coating for medical devices that prevents the attachment of Candida albicans. Ann. Clin. Microbiol. Antimicrob. 16:41. doi: $10.1186 / \mathrm{s} 12941-017-0215-\mathrm{z}$

Vera-González, N., Bailey-Hytholt, C. M., Langlois, L., de Camargo Ribeiro, F., de Souza Santos, E. L., Junqueira, J. C., et al. (2020). Anidulafungin liposome nanoparticles exhibit antifungal activity against planktonic and biofilm Candida albicans. J. Biomed. Mater. Res. A 108, 2263-2276. doi: 10.1002/jbm.a.36984

Welsh, R. M., Bentz, M. L., Shams, A., Houston, H., Lyons, A., Rose, L. J., et al. (2017). Survival, persistence, and isolation of the emerging multidrugresistant pathogenic yeast Candida auris on a plastic health care surface. J. Clin. Microbiol. 55, 2996-3005. doi: 10.1128/JCM.00921-17

Wen, J., Jiang, F., Yeh, C. -K., and Sun, Y. (2016a). Controlling fungal biofilms with functional drug delivery denture biomaterials. Colloids Surf. B 140, 19-27. doi: 10.1016/J.COLSURFB.2015.12.028

Wen, J., Yeh, C. -K., and Sun, Y. (2016b). Functionalized denture resins as drug delivery biomaterials to control fungal biofilms. ACS Biomater. Sci. Eng. 2, 224-230. doi: 10.1021/acsbiomaterials.5b00416

Wen, J., Yeh, C. -K., and Sun, Y. (2018). Salivary polypeptide/hyaluronic acid multilayer coatings act as "fungal repellents" and prevent biofilm formation on biomaterials. J. Mater. Chem. B 6, 1452-1457. doi: 10.1039/ C7TB02592K

Zarnowski, R., Sanchez, H., Covelli, A. S., Dominguez, E., Jaromin, A., Bernhardt, J., et al. (2018). Candida albicans biofilm-induced vesicles confer drug resistance through matrix biogenesis. PLoS Biol. 16:e2006872. doi: 10.1371/journal. pbio. 2006872

Zelikin, A. N. (2010). Drug releasing polymer thin films: new era of surfacemediated drug delivery. ACS Nano 4, 2494-2509. doi: 10.1021/nn100634r

Conflict of Interest: The authors declare that the research was conducted in the absence of any commercial or financial relationships that could be construed as a potential conflict of interest.

Copyright $\odot 2020$ Vera-González and Shukla. This is an open-access article distributed under the terms of the Creative Commons Attribution License (CC BY). The use distribution or reproduction in other forums is permitted, provided the original author(s) and the copyright owner(s) are credited and that the original publication in this journal is cited, in accordance with accepted academic practice. No use, distribution or reproduction is permitted which does not comply with these terms. 